\title{
Modeling User Mobility via User Psychological and Geographical Behaviors Towards Point of-Interest Recommendation
}

\author{
Yan Chen ${ }^{1}, \mathrm{Xin} \mathrm{Li}^{2}$, Lin $\mathrm{Li}^{3}$, Guiquan $\mathrm{Liu}^{1(\bowtie)}$, and Guangdong $\mathrm{Xu}^{4}$ \\ 1 University of Science and Technology of China, Hefei, China \\ \{ycwustc,gqliu\}@utsc.edu.cn \\ 2 iFlyTek Research, Hefei, China \\ xinli2@iflytek.com \\ 3 Wuhan University of Technology, Wuhan, China \\ cathylilin@whut.edu.cn \\ 4 University of Technology Sydney, Sydney, Australia \\ Guandong.Xu@uts.edu.au
}

\begin{abstract}
The pervasive employments of Location-based Social Network call for precise and personalized Point-of-Interest (POI) recommendation to predict which places the users prefer. Modeling user mobility, as an important component of understanding user preference, plays an essential role in POI recommendation. However, existing methods mainly model user mobility through analyzing the check-in data and formulating a distribution without considering why a user checks in at a specific place from psychological perspective. In this paper, we propose a POI recommendation algorithm modeling user mobility by considering check-in data and geographical information. Specifically, with check-in data, we propose a novel probabilistic latent factor model to formulate user psychological behavior from the perspective of utility theory, which could help reveal the inner information underlying the comparative choice behaviors of users. Geographical behavior of all the historical check-ins captured by a power law distribution is then combined with probabilistic latent factor model to form the POI recommendation algorithm. Extensive evaluation experiments conducted on two real-world datasets confirm the superiority of our approach over state-of-the-art methods.
\end{abstract}

Keywords: Location-based social network - Point-of-Interest recommendation · User psychological behavior · Geographical behavior · User mobility

\section{Introduction}

With the prevalence of GPS-enabled portable devices, Location-based Social Networks (LBSNs) have been sweeping the global world during recent years, such as Gowalla, Yelp, Facebook Places, etc. Point-of-Interests (POIs) are places that 
a user may find useful information or tend to visit, e.g. restaurants or shopping malls. As an important component of LBSNs, POI has facilitated an application - POI recommendation. This interesting and useful application can not only benefit merchants by increasing their revenue through virtual marketing but also benefit customers by helping them filter out uninteresting places and reduce their decision making time thus providing satisfactory user experiences [8].

In LBSN, users could check in at a place by sharing their experiences and reviews with friends and online users. Through analyzing the check-in data, researchers can acquire a user's mobility by mining the historical check-in behavior patterns thus giving predictions to her future potential visiting places. Generally, a specific distribution is adopted to formulate a user's mobility, such as multiple Gaussian distribution [1] and power-law distribution [17]. However, such methods have an inherent flaw that they only model the user mobility from the data perspective while without considering the intrinsic reason why a user checks in at a specific place. We argue that user mobility is influenced by user psychological and geographical behaviors. The user psychological behavior can be described on two aspects. The one is when we are confronted with a series of choices, we always compare the utility of these candidates, and are more willing to choose a high utility place to visit [13]. The other is that individual differences influence the user check-in behaviors. Detailedly, a user checked in at a place for ten times and another user only visited the same place thrice. It could not indicate the former prefers the place than the latter, as the former may be a person who likes staying out or traveling and the latter may be a stay-at-home type. Besides, geographical influence matters. We may prefer a nearby place rather than a far one. Although, the distant one is a more popular and higher utility place for us.

Based on the aforementioned summarizes, we give the following assumptions:

- Comparability: the user check-in behavior is a comparative process, where a certain historical check-in place of a user is chosen after he compares the utility of all alternatives rather than a random decision.

- Dissimilarity: the frequency data or the ratings could not give actual expression of user preference. For example, a user visited a place three times while the average visiting time is 3.5 , which indicates the preference to such POI is below the average. However, supposing the same POI visited by another user three times, the average is 2.5. It shows the user prefers the POI more than others. Taking a relative view to address this problem, we adopt partial order relationship deduced from frequency data rather than the frequency value of users' check-in history to learn the user preference.

- Localization: localization is embodied into two aspects. When users arrive at a mart or city, it is believed that they just take the places in the mart or city into account and make a decision. Hence, the candidates are localization, and it motivates us take nearest $\mathrm{K}$ place as our inputs. More importantly, the probability of visiting a POI is inversely proportional to the distance. From an overall perspective, users prefer a nearby place than a distant one and the check-in records are localization. 
In this paper, POI recommendations are made based on the aforementioned assumptions. As demonstrated in Fig. 1, we delve into the mechanism behind the user check-in patterns and propose a probabilistic latent factor model to formulate the user psychological behavior by employing the utility theory of economics which won the Nobel Prize in 2000 [13]. The utility theory conveys that a consumer is more willing to choose those goods that have high utilities to him or her. A power-law distribution is adopted to capture all historical check-in geographical information, also called geographical behavior. To this end, we propose a novel POI recommendation algorithm by combining the user psychological and geographical behaviors to model the user mobility.

In summary, we have made several contributions in this paper:

- We propose a probabilistic latent factor model to mine users psychological behavior from a novel psychological perspective. User mobility is modeling by user psychological and geographical behaviors.

- We devise a stochastic gradient descent algorithm to learn the latent factors of both users and POIs.

- We conduct extensive experiments on two real-world datasets to evaluate our approach. The results demonstrate that our approach outperforms the stateof-the-art methods.

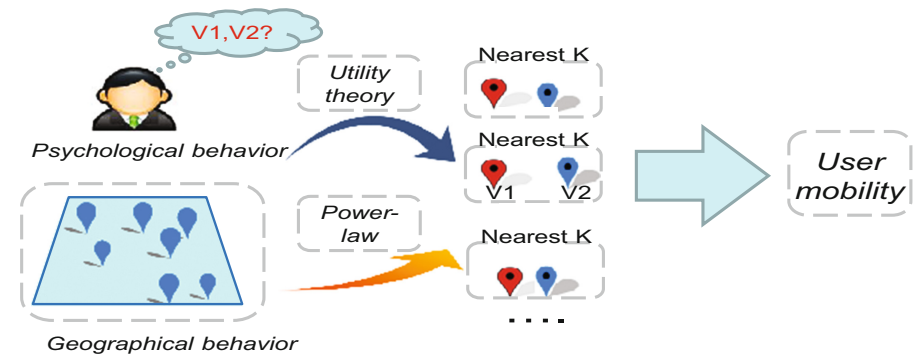

Fig. 1. The framework of the proposed algorithm.

The rest of the paper is organized as follows. We review the recent studies in Sect. 2. The problem formulation and the proposed approach are discussed in Sect. 3. Experiments are presented in Sect. 4. We conclude our work in Sect. 5.

\section{Related Works}

Traditional recommender systems mainly focus on exploiting explicit user-item rating matrix (usually are not available in LBSNs) via employing memory-based [9] or model-based collaborative filtering (CF) $[6,7,11]$. The premise behind memory-based CF is to recommend items by like-minded users of a target user and the intuition of model-based CF is to learn the latent factors e.g. by matrix 
factorization (MF) technology to make recommendations. Moreover, in [3,5], the authors fuse their model with contextual information like social or trust network to improve the prediction accuracy. Although explicit ratings for POIs are not available in LBSNs, traditional CF method can be applied into this filed by treating POIs as common items [16] via exploring user preference from user check-in frequency data which implicitly reflect user preference. Besides, a wide range of properties of LBSN have been extensively explored, such as geographical information $[1,10,12,17,18]$, social connections $[10,17]$, temporal information $[4,8,12]$ and user preference order $[8]$.

As an effective and popular approach to uncover users' preference, modeling user mobility plays an essential role in POI recommendation. Cheng et al. [1] assume that users tend to check in around several centers and model the distance between two locations by the same user as a Multi-center Gaussian Model (MGM) to capture the geographical influence, which is then further into MF for POI recommendation. Ye et al. [17] model user mobility by employing a power-law distribution $(\mathrm{PD})$, and propose a collaborative POI recommendation algorithm based on geographical influence via naive Bayesian. Zhang et al. [18] consider that geographical influence on user mobility should be personalized when LBSNs recommend POIs to users rather than a common distribution for all users, and they model the geographical influence via using kernel density estimation (KDE). Noulas et al. [12] explore the predictive power offered by user mobility features, global mobility features and temporal features, which are finally combined in two supervised learning models. However, these works model user mobility through analyzing the check-in data and formulating the distribution without considering why a user checks in at a specific place from psychological perspective. Intuitively, a certain historical check-in place of a user is chosen under her rational choice to be granted a relatively high utility among all the nearby places.

In this paper, user mobility is captured via geographical and user psychological behaviors. Moreover, user psychological behavior is formulated by probabilistic latent factor model derived from utility theory. In [8,15], the utility theory of economics is adopted to explore the competitive process of user behaviors to make recommendation. However, Li et al. [8] adopt user-POI rating matrix to model choice behavior by adopting time window across recent visits to depict user short-term memory, and Yang et al. [15] take missing values in rating matrix as implicit feedbacks. Our approach simultaneously considers check-in frequency data and geographical information, selecting nearest $\mathrm{K}$ places (both visited and non-visited places) centered at each of users historical check-ins as our inputs. To the best of our knowledge, this work is the first investigation of modeling user mobility from psychological perspective in POI recommendation field.

\section{The Proposed Approach}

In this section, we discuss the problem formulation and propose a method for POI recommendation by modeling the user mobility considering user 
psychological and geographical behaviors. Firstly, we discuss the problem formulation. Then, we mine user psychological behavior using utility theory and propose a probabilistic latent factor model. A stochastic descent algorithm is devised to optimize our proposed model and then we incorporate this model with geographical behavior to formulate user mobility.

To be specific, all the users' check-in places are firstly clustered into several regions according to the clustering phenomenon of Tolbers first law of geography [2]. After that, subsets of each user's historical check-ins are chosen randomly favoring a wide coverage over all the clusters. We then randomly select the nearest $\mathrm{K}$ places (both visited and non-visited places) of each member in the subsets to form several collections which are the inputs of our model. Utility theory is employed to generate the matching degree of the probabilistic latent factors, by leveraging utility of a specific POI to the target user. Through the stochastic gradient descent, we could learn the latent factors of both users and POIs, which depicts the user psychological behavior. Geographical behavior of the historical check-ins of a user is captured by a power-law distribution. Finally, the information of both worlds, i.e. the psychological and geographical behaviors, is then combined thus leading to a final preference score for the future visits. By ranking those scores of a user's un-visited places, we could make recommendation from the top of the list.

\subsection{Problem Formulation}

Suppose that we have $M$ users and $N$ POIs, and let $\mathbb{U}=\left\{u_{1}, u_{2}, \ldots, u_{m}\right\}$ and $\mathbb{V}=\left\{v_{1}, v_{2}, \ldots, v_{n}\right\}$ be a set of users and POIs respectively. Each POI has a location described by longitude and latitude. $f_{u, v}$ denotes the frequency that a user $u$ visited a POI $v$. All the frequency data form a user-POI check-in frequency matrix $F \in \mathbb{R}^{m \times n}$, where 0 denotes non-visited places.

As aforementioned, the nearest $\mathrm{K}$ places of a POI $v_{j}$ (in a diversified subset chosen from multiple clusters) visited by a user $u_{i}$ are chosen to form a collection, termed as a latent POI collection. $C_{i j}^{L}$ denotes a latent POI collection, which means nearest $\mathrm{K}$ places of POI $v_{j}$ visited by user $u_{i}$. In the latent POI collection, the key insight is why the user checked in at a specific place many times, while only visited other places only once or even not visited. As aforementioned, this situation is due to the user psychological behavior and higher frequency indicates more satisfaction of a user to the specific POI. Hence, all POIs in a latent POI collection can be ranged in a partial order relationship w.r.t. their frequencies. It is believed that the partial order relationship implicitly reflects the user psychological behavior.

Definition (POI Partial Order). A partial order in $C_{i j}^{L}$ is shown below:

$$
\succeq_{i j}^{L}=\left\{\hat{v}_{1} \succeq \hat{v}_{2} \succeq \ldots \mid f_{i \hat{v}_{k}} \geq f_{i \hat{v}_{k+1}}, \hat{v}_{k} \in \succeq_{i j}^{L}\right\}
$$

where $f_{i \hat{v}_{k}}$ represents the frequency the user $u_{i}$ visited POI $\hat{v}_{k}$. Figure 2 is a demonstration of POI latent collection and POI partial order collection. 


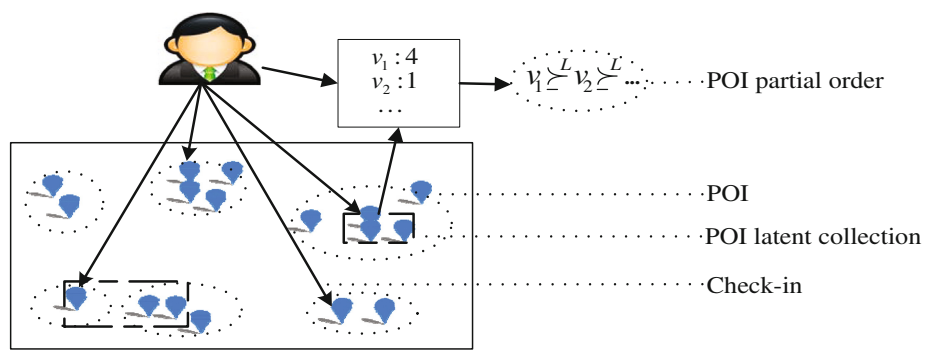

Fig. 2. A demonstration of latent POI collection and POI partial order collection.

\subsection{Modeling User Psychological Behavior}

Generally speaking, it is difficult to quantify the value of a POI, instead, the preference or the psychological behavior of users to the POI can be extracted from the check-in data more readily. Luckily, utility theory defines a kind of measurement of the users' preference over a set of alternatives and could help reveal the inner information underlying the comparative choice behavior of each user [13]. Hence, we use utility theory to model the user psychological behavior in our approach. In this paper, the check-in frequency data are depicted as the utility.

In order to exploit utility theory to model user psychological behavior, we define the utility function $\mathcal{U}(u, v): v \rightarrow \mathbb{R}$ to depict the utility of POI $v$ given by user $u$ in a collection $C^{L}$. Naturally, high utility means more preference of a user to a POI and thus a higher frequency should be granted to the POI. As aforementioned, user psychological behavior implicitly reflected by the relationship between POIs in Eq. (1) becomes meaningful and can be formalized with theory utility. With the partial order in latent collection $C_{i j}^{L}$, the following Eq. (2) reveals user psychological behavior:

$$
v_{j} \succeq v_{j^{\prime}} \operatorname{IIF\mathcal {U}}\left(u_{i}, v_{j}\right) \geq \mathcal{U}\left(u_{i}, v_{j^{\prime}}\right)
$$

which means $v_{j} \succeq v_{j^{\prime}}$ if the utility of $v_{j}$ for $u_{i}$ is larger than that of $v_{j^{\prime}}$.

According to [13], utility usually is decomposed into two parts based on random utility model. Following that is the definition:

$$
\mathcal{U}\left(u_{i}, v_{j}\right)=\mathcal{V}\left(u_{i}, v_{j}\right)+\varepsilon_{i j}
$$

where the first part is the observed utility and the second part is some uncontrollable factors such as emotion, weather or even some occasional events. In our paper, $\mathcal{V}\left(u_{i}, v_{j}\right)$ is depicted as the frequency counted in check-in data, i.e., $\mathcal{V}\left(u_{i}, v_{j}\right)=f_{i j}$. In our approach, we use $f_{i j}=U_{i} V_{j}^{T}$ to parameterize the observed utility [6], where $U_{i} \in \mathbb{R}^{k}, V_{j} \in \mathbb{R}^{k}$ are low-rank determinable latent factors for user $u_{i}$ and item $v_{j}$ respectively and $k$ is the dimension of the latent factor.

The probability of user psychological behavior over alternatives can be defined using the utility of choice [14]:

$$
\operatorname{Pr}\left(v_{j} \succeq v_{j^{\prime}}\right)=\operatorname{Pr}\left(\mathcal{U}\left(u_{i}, v_{j}\right) \geq \mathcal{U}\left(u_{i}, v_{j^{\prime}}\right)\right)
$$


Further, Substitute $\mathcal{U}$ with Eq. (3) and we get the following Eq. (5):

$$
\begin{aligned}
\operatorname{Pr}\left(v_{j} \succeq v_{j^{\prime}}\right) & =\operatorname{Pr}\left(\mathcal{V}\left(u_{i}, v_{j}\right)+\varepsilon_{i j} \geq \mathcal{V}\left(u_{i}, v_{j^{\prime}}\right)+\varepsilon_{i j^{\prime}}\right) \\
& =\operatorname{Pr}\left(\varepsilon_{i j^{\prime}} \leq \varepsilon_{i j}+\mathcal{V}\left(u_{i}, v_{j}\right)-\mathcal{V}\left(u_{i}, v_{j^{\prime}}\right)\right) \\
& =\operatorname{CDF}\left(\varepsilon_{i j}+\mathcal{V}\left(u_{i}, v_{j}\right)-\mathcal{V}\left(u_{i}, v_{j^{\prime}}\right)\right)
\end{aligned}
$$

where $C D F$ is cumulative density function, and the error term is assumed to satisfy $\varepsilon_{i j^{\prime}} \sim$ i.i.d extreme value, that is the double exponential format as $\exp \left(-e^{-\varepsilon}\right)$. The probability for a user psychological behavior over POIs in a latent POI collection is deduced as follows:

$$
\operatorname{Pr}\left(v_{j} \succeq v_{j^{\prime}}\right)=\frac{e^{\mathcal{V}\left(u_{i}, v_{j}\right)}}{\sum_{j^{\prime}}^{\left|C^{L}\right|} e^{\mathcal{V}\left(u_{i}, v_{j^{\prime}}\right)}}
$$

\subsection{Probabilistic Latent Factor Model and Learning Algorithm}

We formulate user psychological behavior for a certain historical check-in place to be granted a relatively utility among all the nearby places and propose a probabilistic latent factor model based on the utility theory. Each POI visited by a user implies a latent collection, so we can easily formulate all users' psychological behaviors and get the probability of whole observation as follows:

$$
\operatorname{Pr}\left(\succeq^{L}\right)=\prod_{u \in \mathbb{U}} \prod_{v_{j} \in \mathbb{V}(u)} \operatorname{Pr}\left(v_{j} \succeq v_{j^{\prime}}\right)=\prod_{u \in \mathbb{U}} \prod_{v_{j} \in \mathbb{V}(u)} \frac{e^{\mathcal{V}\left(u_{i}, v_{j}\right)}}{\sum_{j^{\prime}}^{\left|C^{L}\right|} e^{\mathcal{V}\left(u_{i}, v_{j^{\prime}}\right)}}
$$

As demonstrated above, we use $f_{i j}=U_{i} V_{j}^{T}$ to measure the observed utility, thus assuming a spherical multivariate Gaussian prior on both $U$ and $V$, that is

$$
\operatorname{Pr}(\Omega \mid \Theta)=N\left(\Omega \mid 0, \sigma^{2} I\right)
$$

where $\Omega=\{U, V\}$, and $\Theta$ denotes some hyper-parameters. $\Omega_{l}$ is a component of $\Omega$.

By applying Bayesian theorem, the probabilistic latent factor model can be defined based on the Eqs. (7) and (8).

$$
\operatorname{Pr}(U, V \mid \succeq) \propto \operatorname{Pr}\left(\succeq^{L} \mid U, V\right) \operatorname{Pr}(U \mid \Theta) \operatorname{Pr}(V \mid \Theta)
$$

Given a latent POI collection, to estimate the latent factors, the model can be learned by maximum the posterior (Eq. (9)). For ease of mathematical treatment, we optimize the model using the following equivalent objective function.

$$
\Omega=\arg \min _{\Omega}-\left[\log \operatorname{Pr}\left(\succeq^{L} \mid \Omega\right)+\log \operatorname{Pr}(\Omega \mid \Theta)\right]
$$

where $\log \operatorname{Pr}(\Omega \mid \Theta)$ can be deemed as regularizer $R(\Omega)$ to alleviate overfitting problem. Here, $R(\Omega)$ corresponds to $L 2$ norm regularization.

To learn $U$ and $V$ of the probabilistic latent factor model, a stochastic gradient descent (SGD) algorithm is devised. We can carry out the gradient of above 
object function into two parts respectively, and the first part can be deduced using Eq. (7).

$$
-\prod_{u \in \mathbb{U}} \prod_{v_{j} \in \mathbb{V}(u)} \frac{\partial \log \left(v_{j} \succeq v_{j^{\prime}}\right)}{\partial \Omega_{l}}=\prod_{u \in \mathbb{U}} \prod_{v_{j} \in \mathbb{V}(u)} \frac{\partial\left[\log \left(\sum_{j^{\prime}}^{\left|C^{L}\right|} e^{\mathcal{V}\left(u_{i}, v_{j^{\prime}}\right)}\right)-\mathcal{V}\left(u_{i}, v_{j}\right)\right]}{\partial \Omega_{l}}
$$

The second part can be viewed as:

$$
\frac{\partial \log \operatorname{Pr}(\Omega \mid \Theta)}{\partial \Omega_{l}}=\frac{\partial R(\Omega)}{\partial \Omega_{l}}=\beta \Omega_{l}
$$

where $\beta$ is regularization parameter.

Using $f_{i j}=U_{i} V_{j}^{T}$ to qualify the observed utility, the derivation of $U$ and $V$ can be computed as:

$$
\begin{gathered}
\nabla U_{i}=\frac{\sum_{j \prime}^{\left|C^{L}\right|}\left(e^{U_{i} V_{j^{\prime}}^{T}} \cdot V_{j^{\prime}}\right)}{\sum_{j \prime}^{\left|C^{L}\right|} e^{U_{i} V_{j^{\prime}}^{T}}}-V_{j}+\beta U_{i} \\
\nabla V_{j}=\frac{\left(e^{U_{i} V_{j}^{T}} \cdot U_{i}\right)}{\sum_{j \prime}^{\left|C^{L}\right|} e^{U_{i} V_{j^{\prime}}^{T}}}-U_{i}+\beta V_{j}
\end{gathered}
$$

Given a point, the model parameters are updated by $\Omega_{l} \leftarrow \Omega_{l}-\alpha \nabla \Omega_{l}$ and $\alpha$ is learning rate. Note that we only draw a batch of $\succeq^{L}$ to embed into our learning algorithm.

Now, we have $U$ and $V$ at hand after the optimization is completed above. Hence, the observe utility of a POI $v_{j}$ given by a target user $u_{i}$ can be estimated. Recall the definition of utility, it is a mathematical representation of user psychological behavior, and the predicted utility of a POI $v_{j}$ given by user $u_{i}$ can be denoted:

$$
\operatorname{Pr}[\mathcal{U}(i j)]=\operatorname{Pr}\left(\mathcal{V}(i j)+\varepsilon_{i j}\right)=\mathcal{V}(i j)+\operatorname{Pr}\left(\varepsilon_{i j}\right)=U_{i} V_{j}^{T}+C
$$

where $C$ is a constant.

\subsection{Proposed Algorithm}

We propose a POI recommendation algorithm by modeling user mobility via user psychological and geographical behaviors. As aforementioned, user psychological behavior is captured by utility theory. The geographical behavior measures the probability of whether a user visits a POI under the historical check-in geographical information. In our approach, a power-law distribution is adopted to formulate the geographical behavior [17]. We then formulate the user mobility by fusing the utility with the geographical behavior:

$$
y_{i j} \propto \operatorname{Pr}[\mathcal{U}(i j)] \cdot \operatorname{Pr}\left(v_{j} \mid \mathbb{V}_{i}\right)
$$




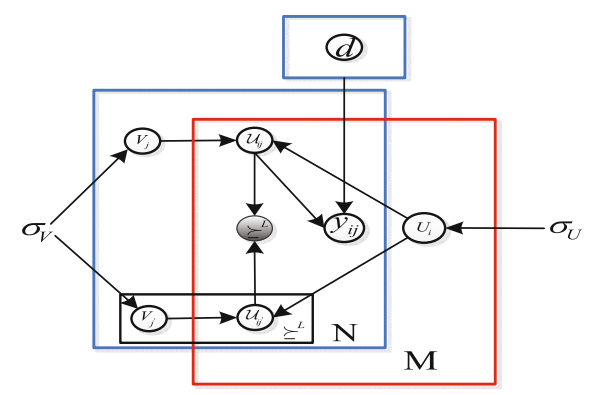

Fig. 3. The graphical representation of the proposed model.

where the $\operatorname{Pr}\left(v_{j} \mid \mathbb{V}_{i}\right)$ is the likelihood probability for the user $u_{i}$ to check at the POI $v_{j} \cdot \operatorname{Pr}\left(v_{j} \mid \mathbb{V}_{i}\right)$ is defined as:

$$
\operatorname{Pr}\left(v_{j} \mid \mathbb{V}_{i}\right)=\prod_{v_{y} \in \mathbb{V}_{i}} \operatorname{Pr}\left[d\left(v_{j}, v_{y}\right)\right]
$$

where $d\left(v_{j}, v_{y}\right)$ denotes the distance between POI $v_{j}$ to $v_{y}$ and $\mathbb{V}_{i}$ is the historical check-in places of user $u_{i}$ and $\operatorname{Pr}\left[d\left(v_{j}, v_{y}\right)\right]=a \times d\left(v_{j}, v_{y}\right)^{b}$.

The graphical model is demonstrated in Fig. 3. The dark circle is the observation variable which denotes the partial order of all POI collections. $\sigma_{U}$ and $\sigma_{V}$ denote hyper-parameters which are placed on $U$ and $V . M$ and $N$ is the size of users and POIs respectively. The blank circle of vector $U_{i}$ and $V_{j}$ are unknown parameters that are learnt by probabilistic latent factor model. After obtaining $U_{i}$ and $V_{j}$, we can estimate the utility of POI $v_{j}$ given by user $u_{i}$. With the utility of $\mathcal{U}_{i j}$ and geographical influence $d$, the preference of user $u_{i}$ to POI $v_{j}$ can be obtained, which is depicted as $y_{i j}$.

As aforementioned, the proposed algorithm can be shown in Algorithm 1.

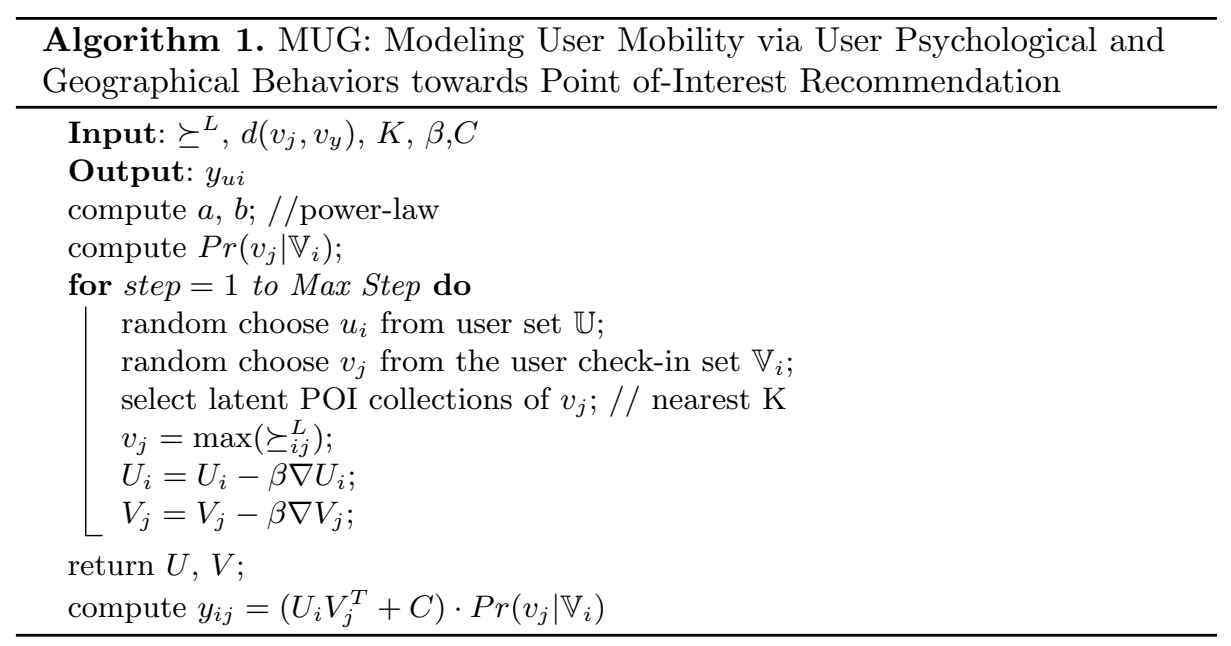




\section{Experiments}

In this section, we compare our model with several state-of-the-art recommendation methods in terms of Information Retrieval (IR) metrics by conducting extensive experiments on two real datasets. We analyze the accuracy of prediction in our POI recommendations. To be specific, we mainly address the following questions: (1) How can we compare our proposed method with existing methods? (2) How the size of latent POI collections influences the results of the proposed method? (3) What are the performances on the cold start problem?

Table 1. Statistics of the two extracted datasets

\begin{tabular}{l|l|l|l}
\hline Datasets & Statistics & User & POI \\
\hline \multirow{2}{*}{ Gowalla } & Max. Num of check-ins & 206 & 308 \\
\cline { 2 - 4 } & Avg. Num of check-ins & 17.03 & 20.19 \\
\hline \multirow{2}{*}{ Brightkite } & Max. Num of check-ins & 140 & 839 \\
\cline { 2 - 4 } & Avg. Num of check-ins & 7.963 & 16.41 \\
\hline
\end{tabular}

\subsection{Datasets and Metrics}

The proposed approach is evaluated on Gowalla ${ }^{1}$ and Brightkite ${ }^{2}$ datasets. Gowalla and Brightkite are location-based social networking service providers where users share their locations, activities and travel lines etc. by checking-in. We randomly extract 3000 users and 2530 POIs with 50724 check-in frequency records from the Gowalla dataset and the density of the extracted dataset is $6.68 \times 10^{-3}$. We randomly extract 5000 users and 2425 POIs with 39815 checkin frequency records from the Brightkite dataset and the density of the extracted dataset is $3.28 \times 10^{-3}$. The statistics of the two datasets are described in Table 1 .

POI recommendation aims to recommend personalized top-N POIs for users, which are obtained after sorting all candidate places in ascending order according to the predicted preference. We use the following three metrics to measure the performance of all models, which are widely adopted in Information Retrieval and Document Classification.

Precision@N and Recall@N: the precision and recall of personalized top-N recommend places for a target user is defined as below:

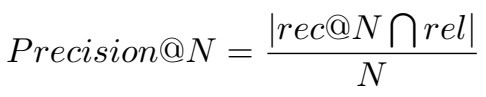

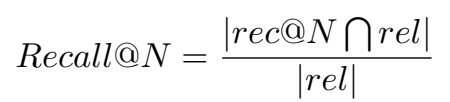

where rec@N denotes the top-N recommended POIs and rel is the true visited POIs in the test data.

\footnotetext{
${ }^{1}$ http://snap.stanford.edu/data/loc-gowalla.html.

2 http://snap.stanford.edu/data/loc-brightkite.html.
} 
MAP: Mean Average Precision (MAP), which for a test collection is the arithmetic mean of average precision values for individual user. The definition is:

$$
M A P=\frac{1}{N} \sum_{u=1}^{M}\left(\frac{1}{r e l} \sum_{l=1}^{|r e l|} \text { Precision }_{u} @ l\right)
$$

where Precision $@ @ l$ denotes the precision for user $u$ when $l$ relevant POIs are retrieved and $M$ is the total number of users.

\subsection{Baseline and Comparison}

We compare our model with the following four existing methods:

- MF: It is adopted in [6], which captures the user preference by factorizing the user-rating matrix to get latent factors.

- PMF: It is proposed in [11] and also places a spherical multivariate Gaussian prior multivariate Gaussian prior on both $U$ and $V$.

- NMF: This method is aimed to find the non-negative matrix factors $U$ and $V[7]$.

- MGM: This algorithm models user check-in behavior via employing Multicenter Gaussian distribution in [1].

- MUG: This is our modeling user mobility via user psychological and geographical behaviors algorithm.

We randomly divide the two extracted datasets into training (80\%) and testing $(20 \%)$ data. The latent dimension is set as 10 for MF, PMF, NMF and our proposed algorithm, following [10]. $\alpha$ and $\beta$ are tuned using 5 fold cross-validation grid search for all algorithms to obtain the best results. $\mathrm{K}$ is set as 20 and 15 for Gowalla dataset and Brightkite dataset respectively in our proposed approach. The comparative experiments repeated by three times, Tables 2, 3 and 4 report the average results of the top 5, top 10 and top 15 POIs on the ranking list, respectively. We can observe that our method outperforms all baseline methods on three metrics. PMF and MGM perform much better than MF and PMF. Moreover,the results are coincident with [10]. Note that the values of precision in Table 2 are low, because the accuracy of POI recommendation is not high on account of sparse datasets. The results are shown detailedly that:

- Compared to the best factor model NMF which does not consider geographical behavior: for instance on top 10, for Gowalla extracted dataset, our method improves the results by $\mathbf{3 3 . 2 0 \%}$ w.r.t. precision, $\mathbf{4 8 . 5 9 \%}$ w.r.t. recall and $\mathbf{2 8 . 7 4} \%$ w.r.t. MAP. As to Brightkite extracted dataset, our method improves the results by $\mathbf{1 3 . 1 2} \%$ w.r.t. precision, $\mathbf{1 6 . 8 4 \%}$ w.r.t. recall and $\mathbf{1 6 . 4 7 \%}$ w.r.t. MAP. It indicates that geographical behavior plays an important role in POI recommendation.

- Compared to the MGM which does not model user mobility from psychological perspective: on top 10, for Gowalla extracted dataset, our method improves the results by $\mathbf{8 2 . 0 9} \%$ on precision, and improves the results by $\mathbf{6 2 . 4 7} \%$ and 
60.55\% on recall and MAP respectively. For Brightkite extracted dataset, our method improves the results by $\mathbf{1 8 1 . 2 9 \%}$ w.r.t. precision, $\mathbf{4 0 . 9 8 \%}$ w.r.t. recall and $\mathbf{9 3 . 8 2} \%$ w.r.t. MAP. MGM considers users tend to check-in around several centers and the historical check-ins follow a Gaussian distribution in every center. However, as the sparse datasets we adopted, the historical checkins may not act up to a multi-center Gaussian distribution. Moreover, MGM is not considering latent factors of user psychological behavior. As a result, our method performs much better than MGM model.

- The results of Gowalla extracted dataset outperform Brightkite extracted dataset because Brightkite extracted dataset is sparser than Gowalla extracted dataset. There is no exception, our proposed algorithm suffers the data sparsity problem, which is one of the most challenging problems in POI recommendations.

Obviously, the proposed approach outperforms the four comparative methods. We attribute the results to the effectiveness of user mobility formulated by user psychological and geographical behaviors in POI recommendation. Besides, the estimated metrics we adopt emphasize the ranking problem which is coincided well with the learning process of our proposed probabilistic latent model. The partial order learning mechanism adopted in our approach works well with the chosen metrics as well, thus making our algorithm superior to state-of-the-art methods.

Table 2. The results of metric precision

\begin{tabular}{l|l|l|l|l|l|l}
\hline Datasets & Pre & MF & PMF & NMF & MGM & MUG \\
\hline Gowalla & $@ 5$ & 0.01659 & 0.01055 & 0.04892 & 0.02641 & $\mathbf{0 . 0 5 2 8 9}$ \\
\cline { 2 - 7 } & $@ 10$ & 0.01627 & 0.00887 & 0.03467 & 0.02536 & $\mathbf{0 . 0 4 6 1 8}$ \\
\cline { 2 - 7 } & $@ 15$ & 0.01088 & 0.00844 & 0.03281 & 0.02283 & $\mathbf{0 . 0 3 9 0 3}$ \\
\hline \multirow{3}{*}{ Brightkite } & $@ 5$ & 0.00740 & 0.00715 & 0.03580 & 0.01138 & $\mathbf{0 . 0 4 7 0 9}$ \\
\cline { 2 - 7 } & $@ 10$ & 0.00742 & 0.00459 & 0.03178 & 0.01278 & $\mathbf{0 . 0 3 5 9 5}$ \\
\cline { 2 - 7 } & $@ 15$ & 0.00820 & 0.00348 & 0.02362 & 0.01218 & $\mathbf{0 . 0 3 0 2 8}$ \\
\hline
\end{tabular}

\subsection{The Cold Start Problem}

The cold start problem is a potential problem in POI recommendation. It may cause the inaccuracy of prediction, as it does not gather abundant information of users or POIs, e.g. new users. To compare our method with comparative algorithms on user cold start problem, we first divide the two datasets into training $(10 \%)$ and testing $(90 \%)$ data and then measure the performances of all models in terms of recall and MAP on top 5, top 10 and top 15. The results are shown in Fig. 4. It is obvious that the performances are different from the results 
Table 3. The results of metric Recall

\begin{tabular}{l|l|l|l|l|l|l}
\hline Datasets & Rec & MF & PMF & NMF & MGM & MUG \\
\hline Gowalla & $@ 5$ & 0.02863 & 0.01107 & 0.12630 & 0.08190 & $\mathbf{0 . 1 4 7 7 5}$ \\
\cline { 2 - 7 } & $@ 10$ & 0.03815 & 0.01637 & 0.16384 & 0.14984 & $\mathbf{0 . 2 4 3 4 6}$ \\
\cline { 2 - 7 } & $@ 15$ & 0.03904 & 0.02893 & 0.21476 & 0.19796 & $\mathbf{0 . 2 5 0 6 6}$ \\
\hline \multirow{3}{*}{ Brightkite } & $@ 5$ & 0.07724 & 0.02350 & 0.08910 & 0.04752 & $\mathbf{0 . 1 0 2 8 9}$ \\
\cline { 2 - 7 } & $@ 10$ & 0.07815 & 0.02122 & 0.12511 & 0.10369 & $\mathbf{0 . 1 4 6 1 9}$ \\
\cline { 2 - 7 } & $@ 15$ & 0.07962 & 0.02922 & 0.16398 & 0.13883 & $\mathbf{0 . 1 9 0 2 5}$ \\
\hline
\end{tabular}

Table 4. The results of metric MAP

\begin{tabular}{l|l|l|l|l|l|l}
\hline Datasets & MAP & MF & PMF & NMF & MGM & MUG \\
\hline Gowalla & $@ 5$ & 0.02144 & 0.01041 & 0.07811 & 0.06477 & $\mathbf{0 . 1 1 5 3 6}$ \\
\cline { 2 - 7 } & $@ 10$ & 0.02239 & 0.01252 & 0.10387 & 0.08329 & $\mathbf{0 . 1 3 3 7 3}$ \\
\cline { 2 - 7 } & $@ 15$ & 0.01857 & 0.01407 & 0.10893 & 0.09210 & $\mathbf{0 . 1 1 1 1 9}$ \\
\hline \multirow{3}{*}{ Brightkite } & $@ 5$ & 0.03913 & 0.02002 & 0.06572 & 0.03751 & $\mathbf{0 . 1 0 6 9 0}$ \\
\cline { 2 - 7 } & $@ 10$ & 0.03783 & 0.02122 & 0.08765 & 0.05267 & $\mathbf{0 . 1 0 2 0 9}$ \\
\cline { 2 - 7 } & $@ 15$ & 0.03968 & 0.02922 & 0.07998 & 0.05554 & $\mathbf{0 . 1 1 5 9 7}$ \\
\hline
\end{tabular}
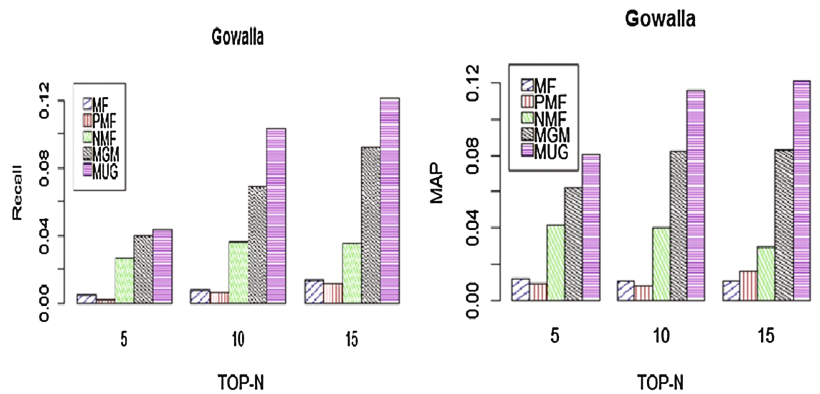

(a) Recall on cold start problem

(b) MAP on cold start problem
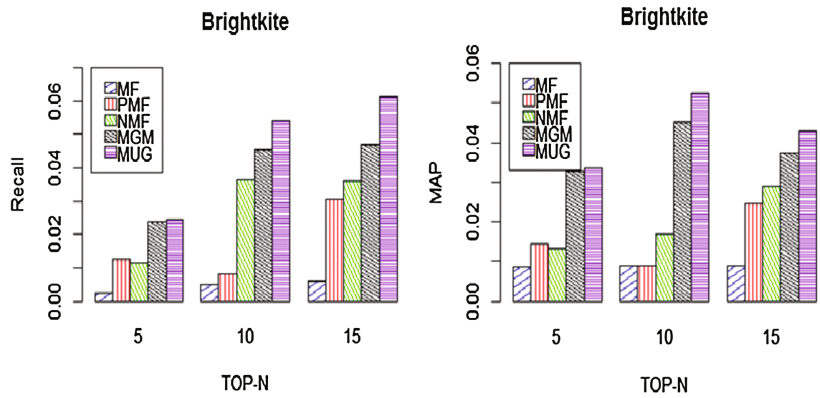

(c) Recall on cold start problem (d) MAP on cold start problem

Fig. 4. Recall and MAP metrics on user cold start problem of both two datasets. 


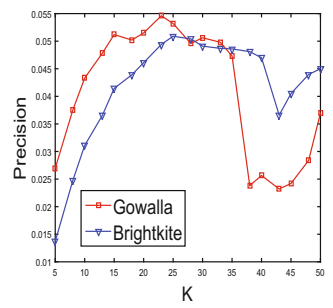

(a) Precision w.r.t. $\mathrm{K}$

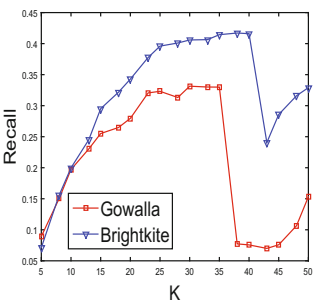

(b) Recall w.r.t. K

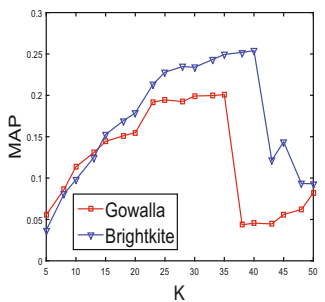

(c) MAP w.r.t. K

Fig. 5. The size of latent POI collections analysis.

of datasets divided into $80 \%$ and $20 \%$. In most cases, our algorithm is superior to baseline algorithms. Moreover, MGM performs better than MF, PMF and NMF, and the results coincide with [1]. From the Fig. 4(a) and (b), on Gowalla datasets, when the check-in records are small and even many users do not have records, we observe that our MUG algorithm outperforms all baseline methods of user cold start problem on both two metrics. As to Brightkite dataset, from the Fig. 4(c) and (d), our MUG algorithm is nearly neck and neck with MGM algorithm on top 5. Luckily, on top 10 and top 15 POIs on the ranking list, MUG algorithm performs better than baseline algorithms. The reason for the good results may lie in user mobility formulated from a psychological perspective. As a whole, our MUG algorithm can improve the accuracy of recommendation on user cold start problem at a certain extent.

\subsection{The Size of Latent POI Collection Analysis}

Here, we analyze the influence of the size of latent POI collections. We analyze the accuracy of top 10 case on both two datasets. $\alpha$ and $\beta$ are set as 0.08 and 0.01 for Gowalla and Brightkite datasets. From the Fig. 5(a), (b) and (c), we can observe several peaks on the line chart which show that our method is sensitive to the size of latent POI collections. For Gowalla dataset, it suggests that when $K \in[10,35]$, our algorithm performs quite well. As to Brightkite dataset, it shows the superiority of $K \in[15,40]$ over other numerical value intervals. The results reveal these $\mathrm{K}$ may be fit well to the size of our datasets we adopted.

\subsection{Impact $\alpha$ and $\beta$}

In our algorithm, the learning rate $\alpha$ controls how quickly the objective function descent and the regularization parameter $\beta$ determines how much the regularization terms should be integrated. From the Fig. 6(a), the results demonstrate that precision negatively correlates with $\alpha$ and achieves the peak when $\alpha=10^{-5}$ on Gowalla and Brightkite extracted datasets. From the Fig. $6(\mathrm{~d})$, as to $\beta$, the results of precision firstly increase with smaller and smaller $\beta$, and they decay when $\beta$ surpasses a certain value, i.e. $10^{-4}$ on our both two datasets. From 
Fig. 6(b), (c), (e) and (f), the results of recall and MAP have the same tendency with the results of precision in terms of $\alpha$ and $\beta$ on the two datasets we used.

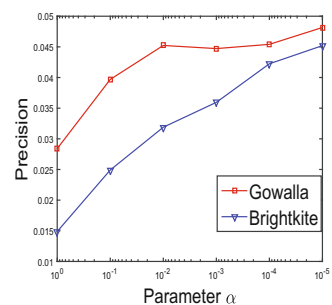

(a) Precision w.r.t. $\alpha$

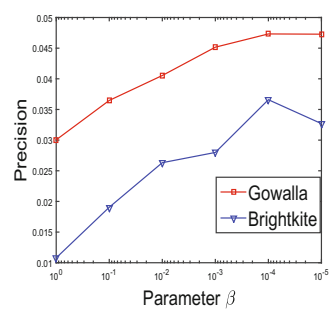

(d) Precision w.r.t. $\beta$

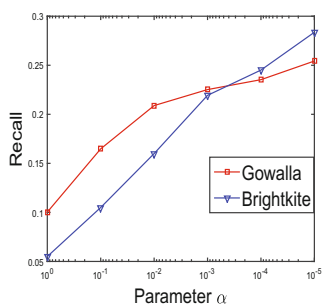

(b) Recall w.r.t. $\alpha$

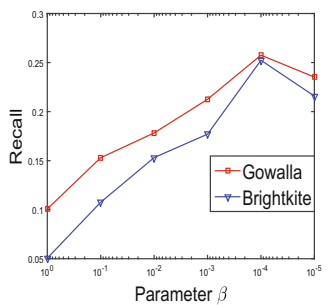

(e) Recall w.r.t. $\beta$

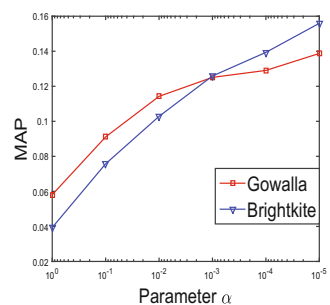

(c) MAP w.r.t. $\alpha$

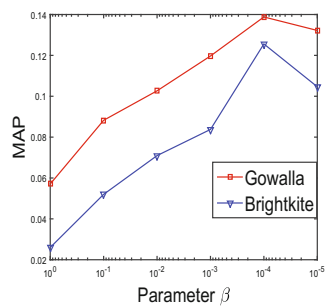

(f) MAP w.r.t. $\beta$

Fig. 6. Impact of parameter $\alpha$ and $\beta$ on three metrics.

\section{Conclusion and Future Work}

In this paper, user psychological and geographical behaviors are combined to model user mobility for POI recommendation. The Proposed probabilistic latent factor model by adopting utility theory is used to formulate user psychological behavior and geographical behavior is captured by adopting a power-law distribution. Besides, a stochastic gradient descent algorithm is devised to learn the probabilistic latent factor model. Our approach is compared with some stateof-the-art POI recommendation algorithms on two real-world datasets and the results demonstrate that our approach achieves better recommendation performance.

For now, we only exploit information from check-in frequency data and geographical information. And data sparsity problem, which is one of the most challenging problems in real-world recommendation scenarios, also inevitably influences the performance of our method. Hence in our future work, there are two directions worthy to study: (1) How to handle with the data sparsity problem such as extremely sparse frequency data? (2) How to fuse leaving information like social networks, temporal information into our model? For future work, it is interesting to incorporate other information, e.g., temporal information, into our model to capture the user interest drift. 
Acknowledgments. This research was partially supported by grants from the Science and Technology Program for Public Wellbeing of China (Grant No. 2013GS340302), National Natural Science Fund Project of China (Grant No. 61232018 and 61325010), National Social and Science Fund project of China (Grant No. 15BGL048), National 863 Plan Project of China (Grant No. 2015AA015403) and Hubei Province Support project of China (Grant No. 2015BAA072).

\section{References}

1. Cheng, C., Yang, H., King, I., Lyu, M.R.: Fused matrix factorization with geographical and social influence in location-based social networks. In: Twenty-Sixth AAAI Conference on Artificial Intelligence (2012)

2. Tobler, W.R.: A computer movie simulating urban growth in the detroit region. Econ. Geogr. 46, 234-240 (1970)

3. Deng, S., Huang, L., Xu, G.: Social network-based service recommendation with trust enhancement. Expert Syst. Appl. 41(18), 8075-8084 (2014)

4. Gao, H., Tang, J., Hu, X., Liu, H.: Exploring temporal effects for location recommendation on location-based social networks. In: Proceedings of the 7th ACM Conference on Recommender Systems, pp. 93-100. ACM (2013)

5. Jamali, M., Ester, M.: Trustwalker: a random walk model for combining trustbased and item-based recommendation. In: Proceedings of the 15th ACM SIGKDD International Conference on Knowledge Discovery and Data Mining, pp. 397-406. ACM (2009)

6. Koren, Y., Bell, R., Volinsky, C.: Matrix factorization techniques for recommender systems. Computer 8, 30-37 (2009)

7. Lee, D.D., Seung, H.S.: Algorithms for non-negative matrix factorization. In: Advances in Neural Information Processing Systems, pp. 556-562 (2001)

8. Li, X., Xu, G., Chen, E., Zong, Y.: Learning recency based comparative choice towards point-of-interest recommendation. Expert Syst. Appl. 42(9), 4274-4283 (2015)

9. Linden, G., Smith, B., York, J.: Amazon.com recommendations: item-to-item collaborative filtering. IEEE Internet Comput. 7(1), 76-80 (2003)

10. Liu, B., Fu, Y., Yao, Z., Xiong, H.: Learning geographical preferences for point-ofinterest recommendation. In: Proceedings of the 19th ACM SIGKDD International Conference on Knowledge Discovery and Data Mining, pp. 1043-1051. ACM (2013)

11. Mnih, A., Salakhutdinov, R.: Probabilistic matrix factorization. In: Advances in Neural Information Processing Systems, pp. 1257-1264 (2007)

12. Noulas, A., Scellato, S., Lathia, N., Mascolo, C.: Mining user mobility features for next place prediction in location-based services. In: 2012 IEEE 12th International Conference on Data Mining (ICDM), pp. 1038-1043. IEEE (2012)

13. Thurstone, L.L.: A law of comparative judgment. Psychol. Rev. 34(4), 273 (1927)

14. Train, K.E.: Discrete Choice Methods with Simulation. Cambridge University Press, New York (2009)

15. Yang, S.H., Long, B., Smola, A.J., Zha, H., Zheng, Z.: Collaborative competitive filtering: learning recommender using context of user choice. In: Proceedings of the 34th International ACM SIGIR Conference on Research and Development in Information Retrieval, pp. 295-304. ACM (2011)

16. Ye, M., Yin, P., Lee, W.C.: Location recommendation for location-based social networks. In: Proceedings of the 18th SIGSPATIAL International Conference on Advances in Geographic Information Systems, pp. 458-461. ACM (2010) 
17. Ye, M., Yin, P., Lee, W.C., Lee, D.L.: Exploiting geographical influence for collaborative point-of-interest recommendation. In: Proceedings of the 34th International ACM SIGIR Conference on Research and Development in Information Retrieval, pp. 325-334. ACM (2011)

18. Zhang, J.D., Chow, C.Y., Li, Y.: igeorec: A personalized and efficient geographical location recommendation framework. IEEE Trans. Serv. Comput. 8(5), 701-714 (2015) 